\title{
Integration of external estimated breeding values and associated reliabilities using correlations among traits and effects
}

\author{
J. Vandenplas, ${ }^{*}{ }^{1,2}$ F. G. Colinet, ${ }^{*}$ G. Glorieux, $\ddagger$ C. Bertozzi, $\ddagger$ and N. Gengler ${ }^{*}$ \\ *Animal Science Unit, Gembloux Agro-Bio Tech, University of Liege, 5030 Gembloux, Belgium \\ †National Fund for Scientific Research, 1000 Brussels, Belgium \\ $\ddagger$ Research and Development Department, Walloon Breeding Association, 5590 Ciney, Belgium
}

\begin{abstract}
Based on a Bayesian view of linear mixed models, several studies showed the possibilities to integrate estimated breeding values (EBV) and associated reliabilities (REL) provided by genetic evaluations performed outside a given evaluation system into this genetic evaluation. Hereafter, the term "internal" refers to this given genetic evaluation system, and the term "external" refers to all other genetic evaluations performed outside the internal evaluation system. Bayesian approaches integrate external information (i.e., external EBV and associated REL) by altering both the mean and (co) variance of the prior distributions of the additive genetic effects based on the knowledge of this external information. Extensions of the Bayesian approaches to multivariate settings are interesting because external information expressed on other scales, measurement units, or trait definitions, or associated with different heritabilities and genetic parameters than the internal traits, could be integrated into a multivariate genetic evaluation without the need to convert external information to the internal traits. Therefore, the aim of this study was to test the integration of external EBV and associated REL, expressed on a 305-d basis and genetically correlated with a trait of interest, into a multivariate genetic evaluation using a random regression test-day model for the trait of interest. The approach we used was a multivariate Bayesian approach. Results showed that the integration of external information led to a genetic evaluation for the trait of interest for, at least, animals associated with external information, as accurate as a bivariate evaluation including all available phenotypic information. In conclusion, the multivariate
\end{abstract}

Received June 1, 2015.

Accepted August 19, 2015.

${ }^{1}$ Corresponding author: jeremie.vandenplas@wur.nl

${ }^{2}$ Current address: Animal Breeding and Genomics Centre, Wageningen UR Livestock Research, $6700 \mathrm{AH}$ Wageningen, the Netherlands.
Bayesian approaches have the potential to integrate external information correlated with the internal phenotypic traits, and potentially to the different random regressions, into a multivariate genetic evaluation. This allows the use of different scales, heritabilities, variance components, measurement units, or trait definitions for external and internal traits. However, one possible issue for implementing multivariate Bayesian approaches could be the availability or estimation of genetic correlations between external and internal traits.

Key words: correlated external information, integration, random regression model

\section{INTRODUCTION}

A combination of phenotypic data into joint evaluations would be theoretically optimal, but often this is not possible for different reasons (Powell and Sieber, 1992). Several studies have shown the possibilities of combining information [i.e., EBV and associated reliabilities (REL); hereafter called "external information"] provided by genetic evaluations performed outside a given evaluation system with genealogical and phenotypic data in a genetic evaluation at an internal level to mimic a joint genetic evaluation. One of the proposed methods is based on a Bayesian view of the linear (mixed) models. This method consists of altering both the mean and (co)variance of the prior distributions of the additive genetic effects based on the knowledge of external information [i.e., based on available external EBV and REL (e.g., Quaas and Zhang, 2006; Legarra et al., 2007; Vandenplas et al., 2014)]. The extension of the Bayesian method to multivariate settings allows the integration of external information into an internal multivariate genetic evaluation (Quaas and Zhang, 2006; Vandenplas et al., 2013). Therefore, the underlying models could be set up to represent different traits for external information and internal phenotypic data exploiting genetic correlations among them in a multivariate setting. Also, the assumed models associated with external information and internal phenotypic data 
could be different from other correlated genetic effects (e.g., regression effects).

Furthermore, the extension to multivariate Bayesian approaches leads them to share the general features and advantages of multivariate genetic evaluations. For example, multivariate evaluations allow the prediction of EBV for traits of interest for which phenotypes could be difficult, or impossible, to collect. They can also improve accuracy of EBV for the different traits under some conditions (Schaeffer, 1994; Mrode, 2005). Thereby, integration of correlated external information into a multivariate genetic evaluation could be interesting to solve different technical issues. First, accuracy of internal evaluations may be low for some traits of interest, whereas accurate external evaluations for similar traits or for correlated traits are routinely performed, potentially at an international level [e.g., multiple acrosscountry evaluations (MACE) for commonly evaluated traits for dairy cattle]. For such evaluations, integration of correlated external information into a multivariate genetic evaluation could improve the accuracy for the traits of interest. Second, external information can be expressed on other scales or measurement units, or it can be associated with different heritabilities, genetic parameters, or trait definitions other than the internal traits. All these issues can lead to genetic correlations below unity.

Our study used the Bayesian approach in the context of dairy cattle production evaluations. In this context, exchanged EBV and REL (e.g., from Interbull, Uppsala, Sweden) are mostly expressed as an average yield on $305 \mathrm{~d}$ for 3 lactations, whereas the underlying genetic evaluations are often based on a (random regression) test-day model (e.g., Auvray and Gengler, 2002). Also, trait definitions and measurement methods for some dairy cattle production traits are not completely harmonized across countries. A good example is the use of different protein traits; most countries use $\mathrm{CP}$, whereas others (e.g., France, United States) use true protein (VanRaden and Powell, 2015). Integration of this type of external information back into underlying genetic evaluations can be considered as a specific case where a multivariate Bayesian approach could be optimal to evaluate genetic merits of animals without the need to convert or deregress external information to be integrated. Therefore, even if the theoretical developments were previously reported, the aim of our study was to test the integration of external information (i.e., EBV and associated REL expressed on a 305-d basis) into a random regression test-day model using correlations among traits and among random regression effects in the context of dairy cattle.

\section{MATERIALS AND METHODS}

\section{Data}

Data, hereafter called "full data set," were provided by the Interreg IVa France-Wallonie-Vlaanderen (Brussels, Belgium) project BlueSel. It consisted of test-day records between 5 and 365 DIM for first-parity, dualpurpose Belgian Blue cows for 2 traits: (1) for protein yield expressed as $\mathrm{CP}(\mathrm{kg})$ and measured in the Walloon Region of Belgium [hereafter called the Belgian trait (B)], and (2) for protein yield expressed as true protein $(\mathrm{kg})$ and measured in France [hereafter called the French trait $(\mathbf{F})]$. Trait $\mathrm{F}$ was assumed to be the trait of interest for our study. In addition, only records of cows which were at least 21 mo old at first calving were selected. After edits, the full data set included 106,606 records for trait B from 14,590 Walloon cows and 38,455 records for trait $\mathrm{F}$ from 5,080 French cows. The pedigree (hereafter called "full pedigree") was also provided by the BlueSel project and consisted of the Belgian and French data combined into a file including 35,978 animals (cows with records and ancestors). The pedigree was traced back to 10 generations starting from the cows with records. A total of 848 bulls sired at least one cow with records in one or in both populations. Among these 848 sires, 104 bulls had progeny in both Belgian and French populations, showing a good genetic link between these 2 populations.

\section{Bivariate Genetic Evaluations}

To be completely independent from differences existing between evaluation methods in routine evaluations in Belgium and France, a harmonized evaluation model was used. The base genetic evaluation model was a bivariate random regression test-day model for traits $\mathrm{B}$ and $\mathrm{F}$. This bivariate model was based on the model used for the Walloon genetic evaluation of production traits (Auvray and Gengler, 2002; Croquet et al., 2006) and was written as

$$
\begin{aligned}
& {\left[\begin{array}{c}
\mathbf{y}_{\mathrm{B}} \\
\mathbf{y}_{\mathrm{F}}
\end{array}\right]=\left[\begin{array}{cc}
\mathbf{X}_{\mathrm{B}} & \mathbf{0} \\
\mathbf{0} & \mathbf{X}_{\mathrm{F}}
\end{array}\right]\left[\begin{array}{c}
\boldsymbol{\beta}_{\mathrm{B}} \\
\boldsymbol{\beta}_{\mathrm{F}}
\end{array}\right]+\left[\begin{array}{cc}
\mathbf{Q}_{\mathrm{B}} & \mathbf{0} \\
\mathbf{0} & \mathbf{Q}_{\mathrm{F}}
\end{array}\right]} \\
& \left(\left[\begin{array}{cc}
\mathbf{Z}_{\mathbf{h B}} & \mathbf{0} \\
\mathbf{0} & \mathbf{Z}_{\mathbf{h F}}
\end{array}\right]\left[\begin{array}{c}
\mathbf{h}_{\mathrm{B}} \\
\mathbf{h}_{\mathrm{F}}
\end{array}\right]+\left[\begin{array}{cc}
\mathbf{Z}_{\mathrm{aB}} & \mathbf{0} \\
\mathbf{0} & \mathbf{Z}_{\mathrm{aF}}
\end{array}\right]\left[\begin{array}{c}
\mathbf{a}_{\mathrm{B}} \\
\mathbf{a}_{\mathrm{F}}
\end{array}\right]+\left[\begin{array}{cc}
\mathbf{Z}_{\mathbf{p B}} & \mathbf{0} \\
\mathbf{0} & \mathbf{Z}_{\mathbf{p F}}
\end{array}\right]\left[\begin{array}{c}
\mathbf{p}_{\mathrm{B}} \\
\mathbf{p}_{\mathrm{F}}
\end{array}\right]\right)+\left[\begin{array}{c}
\mathbf{e}_{\mathrm{B}} \\
\mathbf{e}_{\mathrm{F}}
\end{array}\right],
\end{aligned}
$$

where $\mathbf{y}_{i}(i=\mathrm{B}, \mathrm{F})$ is the vector of observations for the traits $\mathrm{B}$ and $\mathrm{F}$, respectively; $\boldsymbol{\beta}_{i}(i=\mathrm{B}, \mathrm{F})$ is the vector of fixed effects for each trait, $\mathrm{B}$ or $\mathrm{F}$ [i.e., herd 
$\times$ test-day, gestation stage, lactation stage (classes of 5 DIM), and age at calving $\times$ major lactation stage (classes of $73 \mathrm{DIM}) \times$ season of calving]; $\mathbf{h}_{i}(i=\mathrm{B}$, $\mathrm{F})$ is the vector of random herd $\times$ period of calving regression coefficients for each trait (B or $\mathrm{F}) ; \mathbf{a}_{i}(i=\mathrm{B}$, $\mathrm{F})$ is the vector of additive genetic random regression coefficients for each trait ( $\mathrm{B}$ or $\mathrm{F}) ; \mathbf{p}_{i}(i=\mathrm{B}, \mathrm{F})$ is the vector of permanent environmental random regression coefficients for each trait (B or F); and $\mathbf{e}_{i}(i=\mathrm{B}, \mathrm{F})$ is the vector of residuals for each trait $(\mathrm{B}$ or $\mathrm{F})$. The matrices $\mathbf{X}_{i}, \mathbf{Z}_{\mathbf{h} i}, \mathbf{Z}_{\mathbf{a} i}$, and $\mathbf{Z}_{\mathbf{p} i}(i=\mathrm{B}, \mathbf{F})$ were incidence matrices assigning observations to effects, and $\mathbf{Q}_{i}(i=$ $\mathrm{B}, \mathrm{F}$ ) was the covariate matrix for second-order Legendre polynomials.

It was assumed that

$$
\operatorname{Var}\left[\begin{array}{l}
\mathbf{h}_{\mathrm{B}} \\
\mathbf{h}_{\mathrm{F}}
\end{array}\right]=\left[\begin{array}{cc}
\mathbf{I} \otimes \mathbf{H}_{\mathrm{B}} & \mathbf{0} \\
\mathbf{0} & \mathbf{I} \otimes \mathbf{H}_{\mathrm{F}}
\end{array}\right],
$$

where $\mathbf{I}$ is an identity matrix, $\mathbf{H}_{\mathrm{B}}$ and $\mathbf{H}_{\mathrm{F}}$ are the covariance matrices for the random regression coefficients for the herd $\times$ period of calving effect for the traits $B$ and $\mathrm{F}$, respectively, and

$$
\operatorname{Var}\left[\begin{array}{c}
\mathbf{a}_{\mathrm{B}} \\
\mathbf{a}_{\mathrm{F}}
\end{array}\right]=\left[\begin{array}{ll}
\mathbf{A} \otimes \mathbf{G}_{\mathrm{BB}} & \mathbf{A} \otimes \mathbf{G}_{\mathrm{BF}} \\
\mathbf{A} \otimes \mathbf{G}_{\mathrm{FB}} & \mathbf{A} \otimes \mathbf{G}_{\mathrm{FF}}
\end{array}\right],
$$

where $\mathbf{A}$ is the numerator relationship matrix, and

$$
\mathbf{G}=\left[\begin{array}{ll}
\mathbf{G}_{\mathrm{BB}} & \mathbf{G}_{\mathrm{BF}} \\
\mathbf{G}_{\mathrm{FB}} & \mathbf{G}_{\mathrm{FF}}
\end{array}\right]
$$

is the covariance matrix for the random regression coefficients for the additive genetic effects for the traits B and $\mathrm{F}$, respectively. It was also assumed that

$$
\operatorname{Var}\left[\begin{array}{c}
\mathbf{p}_{\mathrm{B}} \\
\mathbf{p}_{\mathrm{F}}
\end{array}\right]=\left[\begin{array}{cc}
\mathbf{I} \otimes \mathbf{P}_{\mathrm{B}} & \mathbf{0} \\
\mathbf{0} & \mathbf{I} \otimes \mathbf{P}_{\mathrm{F}}
\end{array}\right]
$$

where $\mathbf{P}_{\mathrm{B}}$ and $\mathbf{P}_{\mathrm{F}}$ are the covariance matrices for the random regression coefficients for the permanent environmental effects for the traits B and F, respectively, and

$$
\operatorname{Var}\left[\begin{array}{l}
\mathbf{e}_{\mathrm{B}} \\
\mathbf{e}_{\mathrm{F}}
\end{array}\right]=\left[\begin{array}{cc}
\mathbf{I} \sigma_{e \mathrm{~B}}^{2} & \mathbf{0} \\
\mathbf{0} & \mathbf{I} \sigma_{e \mathrm{~F}}^{2}
\end{array}\right],
$$

where $\sigma_{e \mathrm{~B}}^{2}$ and $\sigma_{e \mathrm{~F}}^{2}$ are the residual variances for the traits $\mathrm{B}$ and $\mathrm{F}$, respectively. Estimation of variance components was performed using REMLF90 (Misztal, 2013). For the estimation of variance components, all levels for all fixed and random effects had to be related to at least 5 records. Therefore, the phenotypic data subset used for the estimation of variance components included 63,788 records for the trait B and 20,305 records for the trait $\mathrm{F}$. The associated pedigree included 19,368 animals.

For the computation of EBV, all the following genetic evaluations were performed using the full pedigree (i.e., 35,978 animals) and all the relevant phenotypic records. Three bivariate genetic evaluations were first performed based on the model [1]: (a) a bivariate genetic evaluation (hereafter called "reference evaluation"; $\mathbf{E V A L}_{\mathbf{R}}$ ) using the full phenotypic data set; (b) a bivariate genetic evaluation (hereafter called "Belgian evaluation"; $\mathbf{E V A L}_{\mathbf{B}}$ ) based only on the Belgian records extracted from the full data set; and (c) a bivariate genetic evaluation (hereafter called "French evaluation"; $\mathbf{E V A L}_{\mathbf{F}}$ ) based only on the French records extracted from the full data set. The $\mathrm{EVAL}_{\mathrm{B}}$ and $\mathrm{EVAL}_{\mathrm{F}}$ were equivalent to univariate evaluations including only Belgian and French records, respectively. As defined previously, trait $\mathrm{F}$ was the trait of interest. Therefore, EBV for the trait $\mathrm{F}$ expressed on a $305-\mathrm{d}$ basis $\left(\mathbf{F} 305 ; \mathbf{E B V}_{\mathbf{F}}\right)$ for $\mathrm{EVAL}_{\mathrm{R}}$ and $\mathrm{EVAL}_{\mathrm{F}}$ were computed as

$$
\mathrm{EBV}_{\mathrm{F} j k}=\mathbf{t} \hat{\mathbf{a}}_{\mathrm{F} j k},
$$

where the subscript $j$ refers to the $j$ th evaluation $(j$ $=\mathrm{R}$ and $\mathrm{F}$ for $\mathrm{EVAL}_{\mathrm{R}}$ and $\mathrm{EVAL}_{\mathrm{F}}$, respectively), the subscript $k$ refers to the $k$ th animal, $\mathbf{t}$ is a row vector with the lth element equal to the sum of the lth polynomial from d 1 to 305 , and $\hat{\mathbf{a}}_{\mathrm{F} j k}$ is the vector of the regression coefficients for trait $\mathrm{F}$ for the $k$ th animal. The reliability associated with $\mathrm{EBV}_{\mathrm{F} j k}\left(\mathrm{REL}_{\mathrm{F} j k}\right)$ for the $j$ th evaluation $(j=\mathrm{R}, \mathrm{F})$ and for the $k$ th animal was computed as

$$
\mathrm{REL}_{\mathrm{F} j k}=1-\mathbf{t C}_{j k}^{\mathrm{FF}} \mathbf{t}^{\prime} / \mathbf{t} \mathbf{G}_{\mathrm{FF}} \mathbf{t}^{\prime}
$$

where $\mathbf{C}_{j k}^{\mathrm{FF}}$ is the subset of the inverse of the left-handside of the mixed model equations (MME) corresponding to $\hat{\mathrm{a}}_{\mathrm{F} j k}$. Also, for the $k$ th animal, EBV for trait B expressed on a 305-d basis $\left(\mathbf{B 3 0 5}\right.$; $\left.\mathrm{EBV}_{\mathrm{B}}\right)$ was computed from $\mathrm{EVAL}_{\mathrm{B}}$ as $\mathrm{EBV}_{\mathrm{BB} k}=\mathbf{t a}_{\mathrm{BB} k}$, where $\hat{\mathbf{a}}_{\mathrm{BB} k}$ is the vector of the regression coefficients for the $k$ th animal and for trait $\mathrm{B}$ computed from $\mathrm{EVAL}_{\mathrm{B}}$. The associated REL was computed as $\mathrm{REL}_{\mathrm{BB} k}=1-\mathbf{t C}_{\mathrm{B} k}^{\mathrm{BB}} \mathbf{t}^{\prime} / \mathbf{t G}_{\mathrm{BB}} \mathbf{t}^{\prime}$, where $\mathbf{C}_{\mathrm{B} k}^{\mathrm{BB}}$ is the subset of the inverse of left-hand-side of MME corresponding to $\hat{\mathrm{a}}_{\mathrm{BB} k}$. 


\section{Integration of Correlated External Information}

External information included $\mathrm{EBV}_{\mathrm{BB}}$ and $\mathrm{REL}_{\mathrm{BB}}$ computed from $\mathrm{EVAL}_{\mathrm{B}}$ [i.e., EBV and REL for the trait B305, and (genetically) correlated with trait F] and associated with sires having progeny with Belgian records (hereafter called "external sires"). Based on Vandenplas et al. (2014), a multivariate genetic evaluation (hereafter called "Bayesian evaluation"; $\mathbf{E V A L}_{\mathrm{BA}}$ ) that integrates external information into $\mathrm{EVAL}_{\mathrm{F}}$ was then performed. The model used for $\mathrm{EVAL}_{\mathrm{BA}}$ considered the trait $\mathrm{B} 305$ instead of the trait $\mathrm{B}$ because $\mathrm{EBV}_{\mathrm{BB}}$ and REL $_{\mathrm{BB}}$ were expressed on a 305-d basis. Thereby, the model used for $\mathrm{EVAL}_{\mathrm{BA}}$ was written as follows:

$$
\begin{aligned}
& {\left[\begin{array}{l}
\mathbf{y}_{\mathrm{B}}^{*} \\
\mathbf{y}_{\mathrm{F}}
\end{array}\right]=\left[\begin{array}{cc}
\mathbf{0} & \mathbf{0} \\
\mathbf{0} & \mathbf{X}_{\mathrm{F}}
\end{array}\right]\left[\begin{array}{c}
\mathbf{0} \\
\boldsymbol{\beta}_{\mathrm{F}}
\end{array}\right]+\left[\begin{array}{cc}
\mathbf{I} & \mathbf{0} \\
\mathbf{0} & \mathbf{Q}_{\mathrm{F}}
\end{array}\right]} \\
& \left(\left[\begin{array}{cc}
\mathbf{0} & \mathbf{0} \\
\mathbf{0} & \mathbf{Z}_{\mathbf{h F}}
\end{array}\right]\left[\begin{array}{c}
\mathbf{0} \\
\mathbf{h}_{\mathrm{F}}
\end{array}\right]+\left[\begin{array}{cc}
\mathbf{z}_{\mathrm{aB}}^{*} & \mathbf{0} \\
\mathbf{0} & \mathbf{Z}_{\mathrm{aF}}
\end{array}\right]\left[\begin{array}{c}
\mathbf{a}_{\mathrm{B}}^{*} \\
\mathbf{a}_{\mathrm{F}}
\end{array}\right]+\left[\begin{array}{cc}
\mathbf{0} & \mathbf{0} \\
\mathbf{0} & \mathbf{Z}_{\mathrm{pF}}
\end{array}\right]\left[\begin{array}{c}
\mathbf{0} \\
\mathbf{p}_{\mathrm{F}}
\end{array}\right]\right)+\left[\begin{array}{c}
\mathbf{e}_{\mathrm{B}}^{*} \\
\mathbf{e}_{\mathrm{F}}
\end{array}\right],
\end{aligned}
$$

where $\mathbf{y}_{\mathrm{B}}^{*}$ is the vector of observations for trait B305 corrected for all the effects, except the additive genetic effect; $\mathbf{a}_{\mathrm{B}}^{*}$ is the vector of additive genetic effects expressed on a 305-d basis for the trait B305; and $\mathbf{e}_{\mathrm{B}}^{*}$ is the vector of residuals for the trait B305. The matrix $\mathbf{Z}_{\mathrm{aB}}^{*}$ is an incidence matrix assigning observations to $\mathbf{a}_{\mathrm{B}}^{*}$.

It was assumed that

$$
\begin{gathered}
\operatorname{Var}\left(\mathbf{h}_{\mathrm{F}}\right)=\mathbf{I} \otimes \mathbf{H}_{\mathrm{F}}, \\
\operatorname{Var}\left(\mathbf{p}_{\mathrm{F}}\right)=\mathbf{I} \otimes \mathbf{P}_{\mathrm{F}}, \\
\text { and } \operatorname{Var}\left[\begin{array}{c}
\mathbf{e}_{\mathrm{B}}^{*} \\
\mathbf{e}_{\mathrm{F}}
\end{array}\right]=\left[\begin{array}{cc}
\mathbf{I} \sigma_{e \mathrm{~B}}^{2} & \mathbf{0} \\
\mathbf{0} & \mathbf{I} \sigma_{e \mathrm{~F}}^{2}
\end{array}\right],
\end{gathered}
$$

where $\sigma_{e \mathrm{~B}}^{*_{2}}=\mathbf{t G}_{\mathrm{BB}} \mathbf{t}^{\prime}\left(1-h_{\mathrm{B}}^{2}\right) / h_{\mathrm{B}}^{2}$ is the residual variance for the trait B305 with $h_{\mathrm{B}}^{2}$ being the heritability for trait B305 and estimated from the model [1]. For all these effects, it was assumed that their expectations were equal to 0 . For the additive genetic effects, and following Vandenplas et al. (2014), the expectations of $\left[\begin{array}{c}\mathbf{a}_{\mathrm{B}}^{*} \\ \mathbf{a}_{\mathrm{F}}\end{array}\right]$ were assumed to be equal to

$$
E\left(\begin{array}{c}
\mathbf{a}_{\mathrm{B}}^{*} \\
\mathbf{a}_{\mathrm{F}}
\end{array}\right)=\left[\begin{array}{c}
\boldsymbol{\mu}_{\mathrm{B}} \\
\boldsymbol{\mu}_{\mathrm{F}}
\end{array}\right],
$$

where

$$
\boldsymbol{\mu}_{\mathrm{B}}=\left[\begin{array}{c}
\boldsymbol{\mu}_{s} \\
\mathbf{A}_{o s} \otimes \mathbf{t G}_{\mathrm{BB}} \mathbf{t}^{\prime}\left(\mathbf{A}_{s s} \otimes \mathbf{t G}_{\mathrm{BB}} \mathbf{t}^{\prime}\right)^{-\mathbf{1}} \boldsymbol{\mu}_{s}
\end{array}\right],
$$

with $\boldsymbol{\mu}_{s}$ being the vector of $\mathrm{EBV}_{\mathrm{BB}}$ for external sires, and

$$
\mathbf{A}=\left[\begin{array}{ll}
\mathbf{A}_{s s} & \mathbf{A}_{s o} \\
\mathbf{A}_{o s} & \mathbf{A}_{o o}
\end{array}\right]
$$

where the subscripts $s$ and $o$ refer to the external sires and to the other animals (hereafter called "internal animals"), respectively; and $\boldsymbol{\mu}_{\mathrm{F}}=\mathbf{A} \otimes \mathbf{G}_{\mathrm{FB}} \mathbf{t}^{\prime}\left(\mathbf{A} \otimes \mathbf{t G}_{\mathrm{BB}} \mathbf{t}^{\prime}\right)^{-\mathbf{1}} \boldsymbol{\mu}_{\mathrm{B}}$. The (co)variance matrices for $\left[\begin{array}{c}\mathbf{a}_{\mathrm{B}}^{*} \\ \mathbf{a}_{\mathrm{F}}\end{array}\right]$ were assumed to be equal to

$$
\operatorname{Var}\left(\begin{array}{c}
\mathbf{a}_{\mathrm{B}}^{*} \\
\mathbf{a}_{\mathrm{F}}
\end{array}\right)=\left(\left[\begin{array}{cc}
\mathbf{A} \otimes \mathbf{t G}_{\mathrm{BB}} \mathbf{t}^{\prime} & \mathbf{A} \otimes \mathbf{t G}_{\mathrm{BF}} \\
\mathbf{A} \otimes \mathbf{G}_{\mathrm{FB}} \mathbf{t}^{\prime} & \mathbf{A} \otimes \mathbf{G}_{\mathrm{FF}}
\end{array}\right]^{-\mathbf{1}}+\mathbf{\Lambda}\right)^{-\mathbf{1}},
$$

where $\boldsymbol{\Lambda}$ is a block diagonal (co)variance matrix. Following Vandenplas et al. (2014), the block diagonal elements of $\boldsymbol{\Lambda}$ for the $m$ th external animal were equal to

$$
\boldsymbol{\Lambda}_{m}=\left[\begin{array}{cc}
\operatorname{RE}_{m}\left(\sigma_{e \mathrm{~B}}^{*_{2}}\right)^{-1} & \mathbf{0} \\
\mathbf{0} & \mathbf{0}
\end{array}\right]
$$

where $\mathrm{RE}_{m}$ is equal to the value of records equivalents (RE) only due to own records for trait B305. The block diagonal submatrix equal to $\mathbf{0}$ corresponded to trait $\mathrm{F}$ and zero values were due to the fact that external information was only available for the trait B305. The records equivalents were estimated from $\mathrm{REL}_{\mathrm{BB}}$ thanks to a 2-step algorithm that estimates contributions due to relationships and due to records (Vandenplas and Gengler, 2012). For the internal animals (i.e., animals that are not associated with external information), the corresponding block diagonal elements of $\boldsymbol{\Lambda}$ are equal to $\boldsymbol{\Lambda}_{m}=0$ for the $m$ th internal animal. Similarly to the other evaluations, $\mathrm{EBV}_{\mathrm{F}}$ (i.e., $\mathrm{EBV}$ for the trait F305) for $\mathrm{EVAL}_{\mathrm{BA}}$ were computed using the equations [2] and [3].

It is worth noting that no observations were recorded for trait B305, which led to $\mathbf{y}_{\mathrm{B}}^{*}$ being an empty vector. The contributions for the trait B305 were therefore only provided through external information associated with the external sires. It is also noted that the genetic base associated with $\mathrm{EBV}_{\mathrm{BB}}$ was the original one for the trait B305. However, in practice, EBV are often expressed in function of their own fixed or moving ge- 
netic base; that is, different across evaluations. This issue can be solved by correcting the external EBV before performing the genetic evaluation (Legarra et al., 2007; Vandenplas et al., 2014), or by adding an effect defining the differences among genetic bases of the different evaluations (Legarra et al., 2007). Vandenplas and Gengler (2012) also showed that this effect becomes confounded with the general mean if all external and internal animals are associated with prior information.

The systems of equations for $\mathrm{EVAL}_{\mathrm{R}}, \mathrm{EVAL}_{\mathrm{B}}, \mathrm{EVAL}_{\mathrm{F}}$, and EVAL $_{\mathrm{BA}}$ were solved using BLUPF90 (Misztal, 2013) modified to implement the 2-step algorithm and the Bayesian procedure as proposed by Vandenplas and Gengler (2012) and Vandenplas et al. (2014). Comparisons between $\mathrm{EVAL}_{\mathrm{R}}, \mathrm{EVAL}_{\mathrm{F}}$, and $\mathrm{EVAL}_{\mathrm{BA}}$ were performed separately for (1) external sires with progeny with Belgian or French records (hereafter called "internally used sires"), for (2) external sires without progeny with French records (hereafter called "internally unused sires"), for (3) French progeny sired by external sires and associated with French records, for (4) French progeny sired by external sires and not associated with French records or external information, and for (5) Belgian progeny sired by external sires and not associated with French records or external information. Comparisons were based on (1) Spearman's rank correlation coefficients $\left(\mathbf{r}_{\mathrm{s}}\right)$ of $\mathrm{EBV}_{\mathrm{F}}$ obtained from $\mathrm{EVAL}_{\mathrm{R}}\left(\mathrm{EBV}_{\mathrm{FR}}\right)$ with corresponding $\mathrm{EBV}_{\mathrm{F}}$ obtained from $\operatorname{EVAL}_{\mathrm{F}}\left(\mathrm{EBV}_{\mathrm{FF}}\right)$ or from $\mathrm{EVAL}_{\mathrm{BA}}\left(\mathrm{EBV}_{\mathrm{FBA}}\right)$, (2) regression coefficients (a) of $\mathrm{EBV}_{\mathrm{FR}}$ on $\mathrm{EBV}_{\mathrm{FF}}$ or $\mathrm{EBV}_{\mathrm{FBA}}$, (3) coefficients of determination $\left(\mathbf{R}^{2}\right)$ associated with the regressions, and (4) mean squared errors (MSE) of $\mathrm{EBV}_{\mathrm{FF}}$ and $\mathrm{EBV}_{\mathrm{FBA}}$; MSE were expressed as a percentage of MSE computed for $\mathrm{EBV}_{\mathrm{FF}}$.

\section{RESULTS AND DISCUSSION}

Heritabilities were estimated from the model [1] and expressed on a 305-d basis were 0.48 for the trait B305 and 0.39 for the trait F305. Genetic correlations between B305 and the random genetic regression effects for the trait $\mathrm{F}$ were 0.81 for the intercept, 0.01 for the first order, and -0.04 for the second order. We noted that these genetic correlations included different parts: (1) genetic correlations between traits B and F (confounded with genetic correlations between Belgium and France) and (2) genetic correlations between a constant genetic effect expressed on a 305-d basis and random genetic regression effects. Also, the near zero correlations with linear and quadratic regressions indicated that most of the covariation was between intercepts (i.e., constant genetic effects), as expected.
A total of 617 external sires (i.e., sires with progeny having Belgian records) were associated with external information (i.e., $\mathrm{EBV}_{\mathrm{BB}}$ and $\mathrm{REL}_{\mathrm{BB}}$ computed from EVAL $_{B}$ ). The minimum, average, and maximum $\mathrm{REL}_{\mathrm{BB}}$ associated with these 617 external sires were $>0.00$, 0.38 , and 0.95, respectively. Compared with the rankings of $\mathrm{EVAL}_{\mathrm{F}}$, integration of correlated external information for the 617 external sires led to rankings of EVAL $_{\mathrm{BA}}$ more similar to the rankings of $\mathrm{EVAL}_{\mathrm{R}}$ for all the 5 groups of animals (Table 1). Rank correlations $\mathrm{r}_{\mathrm{s}}$ increased from 0.65 and 0.88 for $\mathrm{EVAL}_{\mathrm{F}}$ to at least 0.99 for $\mathrm{EVAL}_{\mathrm{BA}}$ for the internally used and unused sires, respectively. For French progeny with and without French records, $\mathrm{r}_{\mathrm{s}}$ increased from 0.94 and 0.99 for EVAL $_{F}$, respectively, to at least 0.99 for $\mathrm{EVAL}_{\mathrm{BA}}$. An increase of $r_{s}$ was also observed for the Belgian progeny without French records and external information from 0.68 for $\mathrm{EVAL}_{\mathrm{F}}$ until 0.90 for $\mathrm{EVAL}_{\mathrm{BA}}$ (Table 1).

Furthermore, MSE, a, and $\mathrm{R}^{2}$ also showed that the integration of external information for the 617 external sires into $\mathrm{EVAL}_{\mathrm{F}}$ led to better predictions of $\mathrm{EBV}_{\mathrm{FR}}$ for all 5 groups of animals (Table 1). Therefore, all the 4 parameters (i.e., $\mathrm{r}_{\mathrm{s}}, \mathrm{MSE}$, a, and $\mathrm{R}^{2}$ ) for the 617 external sires showed that the external information correlated with the trait $\mathrm{F}$ is propagated from one trait to the other trait of interest, owing to the correlations among traits and effects (Table 1). Also, according to previous studies, results for the French progeny with or without French records and the Belgian progeny without French records related to the 617 external sires revealed that external information propagated from the 617 external sires to relatives, additionally to from one trait to the other trait (Table 1).

Concerning REL associated with $\mathrm{EBV}_{\mathrm{F}}$ obtained from the different evaluations (i.e., $\mathrm{EVAL}_{\mathrm{BA}}, \mathrm{EVAL}_{\mathrm{F}}$, and $\mathrm{EVAL}_{\mathrm{R}}$ ), an increase of average REL after the integration of external information was observed for all the 5 groups of animals. For the internally used sires, average REL increased from 0.42 for $\mathrm{EVAL}_{\mathrm{F}}$ until 0.55 for $E_{V A L}$. The average REL for $E V_{A} L_{R}$ was equal to 0.56 (Table 1). Therefore, almost all contributions observed in $\mathrm{EVAL}_{\mathrm{R}}$ for the internally used sires were recovered after the integration of external information. Considering the internally unused sires, the average REL increased from 0.06 for $\mathrm{EVAL}_{\mathrm{F}}$ until 0.25 for EVAL $_{\mathrm{BA}}$ (i.e., equal to the average REL for $\mathrm{EVAL}_{\mathrm{R}}$; Table 1). These results associated with the 4 parameters (i.e., $\mathrm{r}_{\mathrm{s}}, \mathrm{a}, \mathrm{R}^{2}$, and MSE) showed that all the Belgian contributions were recovered, which led to a more reliable genetic evaluation for these sires. For the French progeny with and without French records, the average REL increased from 0.48 and 0.28 , respectively, for $\mathrm{EVAL}_{\mathrm{F}}$ until 0.49 and 0.30 for $\mathrm{EVAL}_{\mathrm{BA}}$. These re- 
Table 1. Average reliabilities (REL) associated with estimated breeding values for the French trait expressed on a 305-d basis (EBV $\mathrm{F}_{\mathrm{F}}$ and parameters ${ }^{1}$ obtained for the 5 groups of animals and for the reference, French, and Bayesian evaluations

\begin{tabular}{|c|c|c|c|c|c|c|c|}
\hline \multirow[b]{2}{*}{ Group of animals } & \multirow[b]{2}{*}{ Number } & \multirow[b]{2}{*}{ Evaluations } & \multirow[b]{2}{*}{ REL (SD) } & \multicolumn{4}{|c|}{ Parameter $^{1}$} \\
\hline & & & & $\mathrm{r}_{\mathrm{s}}$ & $\% \mathrm{MSE}$ & a & $\mathrm{R}^{2}$ \\
\hline \multirow{2}{*}{ Internally used sires } & \multirow{2}{*}{104} & French & $0.42(0.28)$ & 0.88 & 100.00 & 0.73 & 0.78 \\
\hline & & Bayesian & $0.55(0.24)$ & 0.99 & 4.50 & 0.99 & 0.99 \\
\hline \multirow[t]{2}{*}{ Internally unused sires } & \multirow[t]{2}{*}{513} & Reference & $0.25(0.16)$ & - & - & - & - \\
\hline & & French & $0.06(0.08)$ & 0.65 & 100.00 & 0.34 & 0.43 \\
\hline \multirow{2}{*}{ French progeny with French records } & \multirow{2}{*}{1,681} & French & $0.48(0.09)$ & 0.99 & 100.00 & 0.99 & 0.98 \\
\hline & & Bayesian & $0.49(0.09)$ & $>0.99$ & 11.12 & 1.00 & $>0.99$ \\
\hline \multirow[t]{3}{*}{ French progeny without French records and information } & \multirow[t]{3}{*}{238} & Reference & $0.30(0.14)$ & - & - & - & - \\
\hline & & French & $0.28(0.14)$ & 0.94 & 100.00 & 0.86 & 0.88 \\
\hline & & Bayesian & $0.30(0.14)$ & $>0.99$ & 8.22 & 0.98 & 0.99 \\
\hline \multirow[t]{2}{*}{ Belgian progeny without French records and information } & \multirow[t]{2}{*}{1,314} & Reference & $0.16(0.10)$ & - & - & - & - \\
\hline & & French & $0.07(0.08)$ & 0.68 & 100.00 & 0.38 & 0.46 \\
\hline
\end{tabular}

${ }^{1} \mathrm{r}_{\mathrm{s}}=$ Spearman's rank correlation coefficients of $\mathrm{EBV}_{\mathrm{F}}$ obtained from the reference evaluation $\left(\mathrm{EBV}_{\mathrm{FR}}\right)$ with corresponding EBV $\mathrm{F}_{\mathrm{F}}$ obtained from the French evaluation $\left(\mathrm{EBV}_{\mathrm{FF}}\right)$ or from the Bayesian evaluation $\left(\mathrm{EBV}_{\mathrm{FBA}}\right)$; $\mathrm{a}=$ regression coefficients of $\mathrm{EBV}_{\mathrm{FR}}$ on $\mathrm{EBV}_{\mathrm{FF}}$ or $\mathrm{EBV}_{\mathrm{FBA}}$; $\mathrm{R}^{2}=$ coefficients of determination associated with the regressions; \% $\mathrm{MSE}=$ mean squared errors of $\mathrm{EBV}_{\mathrm{FF}}$ and $\mathrm{EBV} \mathrm{FBA}_{\mathrm{FB}}$ expressed as a percentage of MSE obtained for $\mathrm{EBV}_{\mathrm{FF}}$.

sults for French progeny could be expected because most of the contributions were already represented in $\mathrm{EVAL}_{\mathrm{F}}$. It was also worth noting that average REL for Belgian progeny without French records and external information increased from 0.07 for $\mathrm{EVAL}_{\mathrm{F}}$ until 0.12 for $\mathrm{EVAL}_{\mathrm{BA}}$. The corresponding average REL for EV$\mathrm{AL}_{\mathrm{R}}$ was 0.16 . The difference in REL between $\mathrm{EVAL}_{\mathrm{BA}}$ and $\mathrm{EVAL}_{\mathrm{R}}$ can be attributed to the fact that the only contributions for this group of animals originated from the contributions associated with their external sires. Also, the propagation of external information must be performed from the external sires to their Belgian progeny and from trait B305 to trait F.

Based on these results, the multivariate Bayesian approaches (e.g., Vandenplas et al., 2014) allowed the combination of different sources of information associated with different scales, different heritabilities and variance components, different measurement units, and different trait definitions between the internal and external evaluations. Also, although it was not discussed in our study, these approaches allow considering genotype by environment interactions based on correlations below unity for the same traits in different environments. All these characteristics permit to avoid conversion or deregression of external information (e.g., expressed as indices or standardized estimates) to the expression of the random animal effects considered by the internal evaluations. This is especially interesting in the context of dairy cattle evaluations, in which EBV and REL are mostly expressed as an average yield on $305 \mathrm{~d}$ for several lactations (Gengler and Vanderick, 2008; Liu et al., 2014). Similarly to our study, such external information could be integrated into a genetic evaluation based on a test-day model as an additional trait which has no observed phenotype internally and which is genetically correlated with all the additive genetic effects for all lactations. Also, such a strategy could be considered for integrating MACE information into single-step genomic evaluations (Misztal et al., 2009; Christensen and Lund, 2010) based on test-day models. An additional application of such a strategy concerns the genetic evaluations for novel traits. Indeed, even in the most advanced countries, phenotypes for novel traits are still sparse (e.g., FA), or their trait definitions (e.g., for feed intake, methane) are very unstable (e.g., Gengler et al., 2010; Manzanilla Pech et al., 2014). Therefore, optimal use of information from correlated traits (e.g., indirect predictors, different trait definitions), potentially also available through MACE, could be useful.

\section{CONCLUSIONS}

The multivariate Bayesian approaches have the potential to integrate external information correlated with the internal phenotypic traits and, potentially to different random regressions, into a multivariate genetic evaluation. This allows the use of different scales, heritabilities, variance components, measurement units, or trait definitions between external and internal traits. However, one possible issue for implementing the proposed approaches could be the availability or estimation of genetic correlations between external information and internal data. 


\section{ACKNOWLEDGMENTS}

Jérémie Vandenplas performed this research as a research fellow, and Nicolas Gengler as a former senior research associate. Both acknowledge the support of their funding agency, the National Fund for Scientific Research (Brussels, Belgium). The authors acknowledge the Institut de l'Elevage (Paris, France) for providing the French data. This research was partially supported by the European Union (European Regional Development Fund) and the Walloon Region of Belgium (SPW-DGO3 Agriculture, Natural Resources and Environment), in particular through the Interreg IVa France-Wallonie-Vlaanderen project BlueSel. The authors thank the anonymous reviewers and the section editor for their useful comments.

\section{REFERENCES}

Auvray, B., and N. Gengler. 2002. Feasibility of a Walloon test-day model and study of its potential as tool for selection and management. Interbull Bull. 29:123-127.

Christensen, O. F., and M. S. Lund. 2010. Genomic prediction when some animals are not genotyped. Genet. Sel. Evol. 42:2.

Croquet, C., P. Mayeres, A. Gillon, S. Vanderick, and N. Gengler. 2006. Inbreeding depression for global and partial economic indexes, production, type, and functional traits. J. Dairy Sci. 89:2257-2267.

Gengler, N., and S. Vanderick. 2008. Bayesian inclusion of external evaluations into a national evaluation system: Application to milk production traits. Interbull Bull. 38:70-74.

Gengler, N., S. Vanderick, V. Arnould, and H. Soyeurt. 2010. Genetic evaluation for milk fat composition in the Walloon Region of Belgium. Interbull Bull. 42:81-84.

Legarra, A., J. K. Bertrand, T. Strabel, R. L. Sapp, J. P. Sanchez, and I. Misztal. 2007. Multi-breed genetic evaluation in a Gelbvieh population. J. Anim. Breed. Genet. 124:286-295.
Liu, Z., M. E. Goddard, F. Reinhardt, and R. Reents. 2014. A singlestep genomic model with direct estimation of marker effects. J. Dairy Sci. 97:5833-5850.

Manzanilla Pech, C. I. V., R. F. Veerkamp, M. P. L. Calus, R. Zom, A. van Knegsel, J. E. Pryce, and Y. De Haas. 2014. Genetic parameters across lactation for feed intake, fat- and protein-corrected milk, and liveweight in first-parity Holstein cattle. J. Dairy Sci. 97:5851-5862.

Misztal, I. 2013. BLUPF90 family of programs. Accessed May 22, 2015. http://nce.ads.uga.edu/wiki/doku.php.

Misztal, I., A. Legarra, and I. Aguilar. 2009. Computing procedures for genetic evaluation including phenotypic, full pedigree, and genomic information. J. Dairy Sci. 92:4648-4655.

Mrode, R. A. 2005. Linear Models for the Prediction of Animal Breeding Values. 2nd ed. CABI Publishing, Wallingford, UK.

Powell, R. L., and M. Sieber. 1992. Direct and indirect conversion of bull evaluations for yield traits between countries. J. Dairy Sci. $75: 1138-1146$

Quaas, R. L., and Z. Zhang. 2006. Multiple-breed genetic evaluation in the US beef cattle context: Methodology. CD-ROM Comm. 24-12 in Proceedings of the 8th World Congress on Genetics Applied to Livestock Production, Belo Horizonte, Brazil. WCGALP, Belo Horizonte, Brazil.

Schaeffer, L. R. 1994. Multiple-country comparison of dairy sires. J. Dairy Sci. 77:2671-2678.

Vandenplas, J., F. G. Colinet, and N. Gengler. 2014. Unified method to integrate and blend several, potentially related, sources of information for genetic evaluation. Genet. Sel. Evol. 46:59.

Vandenplas, J., and N. Gengler. 2012. Comparison and improvements of different Bayesian procedures to integrate external information into genetic evaluations. J. Dairy Sci. 95:1513-1526.

Vandenplas, J., S. Janssens, N. Buys, and N. Gengler. 2013. An integration of external information for foreign stallions into the Belgian genetic evaluation for jumping horses. J. Anim. Breed. Genet. 130:209-217.

VanRaden, P. M., and R. L. Powell. 2015. Genetic evaluations for true protein. Accessed May 22, 2015. https://aipl.arsusda.gov/ reference/trueprot.htm. 